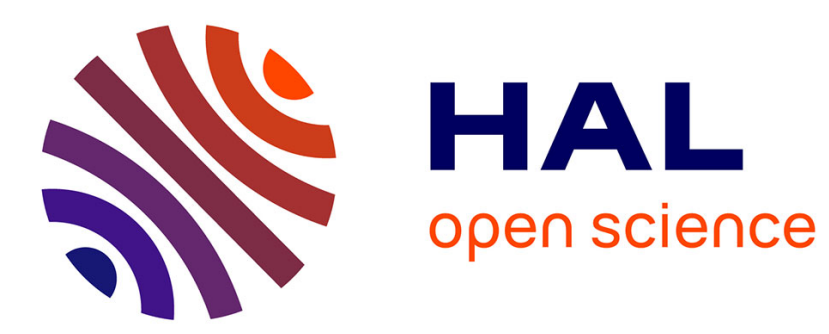

\title{
Observability conservation by output feedback and observability Gramian bounds
}

\author{
Liangquan Zhang, Qinghua Zhang
}

\section{To cite this version:}

Liangquan Zhang, Qinghua Zhang. Observability conservation by output feedback and observability Gramian bounds. Automatica, 2015, 60, pp.5. 10.1016/j.automatica.2015.06.031 . hal-01231786

\section{HAL Id: hal-01231786 \\ https://inria.hal.science/hal-01231786}

Submitted on 20 Nov 2015

HAL is a multi-disciplinary open access archive for the deposit and dissemination of scientific research documents, whether they are published or not. The documents may come from teaching and research institutions in France or abroad, or from public or private research centers.
L'archive ouverte pluridisciplinaire HAL, est destinée au dépôt et à la diffusion de documents scientifiques de niveau recherche, publiés ou non, émanant des établissements d'enseignement et de recherche français ou étrangers, des laboratoires publics ou privés. 


\title{
Observability conservation by output feedback and observability Gramian bounds
}

\author{
Liangquan Zhang ${ }^{\mathrm{a}}$, Qinghua Zhang ${ }^{\mathrm{a}}$, \\ ${ }^{\mathrm{a}}$ INRIA, Campus de Beaulieu, 35042 Rennes, France
}

\begin{abstract}
Though it is a trivial fact that the observability of a linear state space system is conserved by output feedback, it requires a rigorous proof to generalize this result to uniform complete observability, which is defined with the observability Gramian. The purpose of this paper is to present such a proof. Some issues in existing results are also discussed. The uniform complete observability of closed loop systems is useful for the analysis of some adaptive systems and of the Kalman filter.
\end{abstract}

Key words: observability Gramian, uniform complete observability, time varying system, closed loop system.

\section{Introduction}

For a linear time varying (LTV) state space system

$$
\begin{aligned}
\dot{x}(t) & =A(t) x(t)+B(t) u(t) \\
y(t) & =C(t) x(t)
\end{aligned}
$$

with the state $x(t) \in \mathbb{R}^{n}$, the input $u(t) \in \mathbb{R}^{k}$, the output $y(t) \in \mathbb{R}^{m}$, and with bounded piecewise continuous matrices $A(t), B(t), C(t)$ of appropriate sizes, it is well known that its observability depends only on the matrix pair $[A(t), C(t)]$.

Consider the output feedback $u(t)=-L(t) y(t)$ with some matrix $L(t) \in \mathbb{R}^{k \times m}$ and let $K(t) \triangleq B(t) L(t) \in$ $\mathbb{R}^{n \times m}$, then the closed loop system

$$
\begin{aligned}
\dot{x}(t) & =A(t) x(t)-K(t) y(t) \\
y(t) & =C(t) x(t)
\end{aligned}
$$

and the equivalent system

$$
\begin{aligned}
& \dot{x}(t)=(A(t)-K(t) C(t)) x(t) \\
& y(t)=C(t) x(t) .
\end{aligned}
$$

have the same observability property as (1).

It is then clear that, if the matrix pair $[A(t), C(t)]$ is observable, then so is the matrix pair $[(A(t)-$ $K(t) C(t)), C(t)]$. The converse is also true. In (Anderson et al., 1986, page 38), the statement of this result was generalized to uniform complete observability (UCO, definition recalled in the next section), but without being proved.

Proofs of the generalized result can be found in (Sastry

\footnotetext{
$\star$ This work has been supported by the ITEA MODRIO project.

Email addresses: Liangquan. Zhang@inria.fr (Liangquan Zhang), Qinghua.Zhang@inria.fr (Qinghua Zhang).
}

and Bodson, 1989; Ioannou and Sun, 1996), but some details of these proofs merit revision and comments, as discussed in Appendix A. The purpose of this paper is to propose a complete proof with new observability Gramian bounds.

In (Aeyels et al., 1998) a similar result is presented, but it does not provide an estimation of the bounds of the observability Gramian, and assumes an extra condition: the uniform stability of the considered systems, which is not required in the present paper.

Matrices of the form $(A(t)-K(t) C(t))$ appear naturally in the error dynamics equation of the Kalman filter, and similarly in state observers. The UCO of the matrix pair $[(A(t)-K(t) C(t)), C(t)]$ helps to establish the stability of the Kalman filter or of state observers (Kalman, 1963). It is also useful for the analysis of some adaptive systems (Anderson et al., 1986, chapter 2), (Sastry and Bodson, 1989, chapter 2), (Ioannou and Sun, 1996, chapter 4).

\section{Observability Gramian bounds}

Let $\Phi\left(t, t_{0}\right)$ denote the state transition matrix of system (1). The observability Gramian of this system is

$$
M\left(t_{1}, t_{2}\right)=\int_{t_{1}}^{t_{2}} \Phi^{T}\left(t, t_{2}\right) C^{T}(t) C(t) \Phi\left(t, t_{2}\right) \mathrm{d} t .
$$

System (1) is said uniformly completely observable $\left(\mathrm{UCO}^{1}\right.$ ) (Kalman, 1963, p. 358) if there exist positive constants $\sigma, \alpha, \beta$ such that $\left(I_{n}\right.$ denotes the $n \times n$ identity

\footnotetext{
1 In this paper "UCO" is used either as a noun or as an adjective. Some variants of the definition exist (Sastry and Bodson, 1989; Ioannou and Sun, 1996). The definition recalled here follows (Kalman, 1963).
} 
matrix)

$$
0<\alpha I_{n} \leq M(t-\sigma, t) \leq \beta I_{n}
$$

hold for all $t \in \mathbb{R}$. Similarly, let $\tilde{\Phi}\left(t, t_{0}\right)$ be the state transition matrix of system (3), and its observability Gramian

$$
\tilde{M}\left(t_{1}, t_{2}\right)=\int_{t_{1}}^{t_{2}} \tilde{\Phi}^{T}\left(t, t_{2}\right) C^{T}(t) C(t) \tilde{\Phi}\left(t, t_{2}\right) \mathrm{d} t .
$$

System (3) is said UCO if there exist positive constants $\tilde{\sigma}, \tilde{\alpha}, \tilde{\beta}$ such that, for all $t \in \mathbb{R}$,

$$
0<\tilde{\alpha} I_{n} \leq \tilde{M}(t-\tilde{\sigma}, t) \leq \tilde{\beta} I_{n} .
$$

The following result was stated in (Anderson et al., 1986, p. 38, Lemma 2.3).

Lemma 1 The matrix pair $[A(t), C(t)]$ is $U C O$, if and only if for any bounded and locally integrable matrix $K(t)$ the matrix pair $[(A(t)-K(t) C(t)), C(t)]$ is $U C O$.

For a rigorous proof of this result, it should be shown that the existence of $\sigma, \alpha, \beta$ implies the existence of $\tilde{\sigma}, \tilde{\alpha}, \tilde{\beta}$, and vice versa. No such proof was given in (Anderson et al., 1986). The same lemma (up to minor differences) is presented in (Sastry and Bodson, 1989; Ioannou and Sun, 1996) with similar proofs, but some details merit revision and comments, as discussed in Appendix A.

Throughout this paper, "\|· $\|$ " will denote the Euclidean vector norm or the matrix norm induced by the Euclidean vector norm.

Let us prove the following more complete result.

Lemma 2 Assume that the positive constants $\gamma, \eta, \rho$ are such that the inequalities

$$
\|A(t)\| \leq \gamma,\|C(t)\| \leq \eta,\|K(t)\| \leq \rho
$$

hold for all $t \in \mathbb{R}$, and that the observability Gramian of the matrix pair $[A(t), C(t)]$ satisfies the inequalities (5) with some positive constants $\sigma, \alpha, \beta$, then the observability Gramian of the matrix pair $[(A(t)-K(t) C(t)), C(t)]$ satisfies the inequalities (7) with $\tilde{\sigma}=\sigma$ and

$$
\begin{aligned}
& \tilde{\alpha}=\left\{\begin{array}{cl}
\left(\frac{\sqrt{\beta-\alpha+\varphi \alpha}-\sqrt{\beta}}{\varphi-1}\right)^{2}>0 & \text { if } \varphi \neq 1 \\
\frac{\alpha^{2}}{4 \beta}>0 & \text { if } \varphi=1
\end{array}\right. \\
& \tilde{\beta}=(\sqrt{\beta-\alpha+\psi \beta}+\sqrt{\beta})^{2}
\end{aligned}
$$

where

$$
\begin{aligned}
& \varphi=\eta^{2} \rho^{2}\left(\frac{e^{2 \gamma \sigma}-1}{4 \gamma^{2}}-\frac{\sigma}{2 \gamma}\right) \\
& \psi=\eta^{2} \rho^{2}\left(\frac{e^{2(\gamma+\eta \rho) \sigma}-1}{4(\gamma+\eta \rho)^{2}}-\frac{\sigma}{2(\gamma+\eta \rho)}\right) .
\end{aligned}
$$

Notice the "symmetry" between $A(t)$ and

in the sense that

$$
\tilde{A}(t) \triangleq A(t)-K(t) C(t)
$$

$$
A(t)=\tilde{A}(t)-(-K(t)) C(t),
$$

hence the converse of Lemma 2 is implied by the lemma itself.

The proof of Lemma 2 will need the following result.

Lemma 3 Let $\Phi\left(t, t_{0}\right)$ be the state transition matrix of $\dot{x}(t)=A(t) x(t)$ with $\|A(t)\| \leq \gamma$, then $\left\|\Phi\left(t, t_{0}\right)\right\| \leq$ $e^{\gamma\left|t-t_{0}\right|}$ for all $t, t_{0} \in \mathbb{R}$.

See (Chicone, 2006), Theorem 2.4. for a proof of this result.

\section{Proof of Lemma 2}

Let us first establish the relationship between $\Phi\left(t, t_{0}\right)$ and $\tilde{\Phi}\left(t, t_{0}\right)$.

The solution of $(3 \mathrm{a})$, with some initial state $x\left(t_{0}\right)$, is

$$
x(t)=\tilde{\Phi}\left(t, t_{0}\right) x\left(t_{0}\right) .
$$

On the other hand, rewrite $(3 \mathrm{a})$ as

$$
\dot{x}(t)=A(t) x(t)-K(t) C(t) x(t)
$$

and treat $-K(t) C(t) x(t)=-K(t) C(t) \tilde{\Phi}\left(t, t_{0}\right) x\left(t_{0}\right)$ as the input term, then

$x(t)=\Phi\left(t, t_{0}\right) x\left(t_{0}\right)-\int_{t_{0}}^{t} \Phi(t, p) K(p) C(p) \tilde{\Phi}\left(p, t_{0}\right) x\left(t_{0}\right) \mathrm{d} p$.

For any initial state $x\left(t_{0}\right) \in \mathbb{R}^{n}$, the right hand sides of (14) and of (16) are equal, hence

$$
\tilde{\Phi}\left(t, t_{0}\right)=\Phi\left(t, t_{0}\right)-\int_{t_{0}}^{t} \Phi(t, p) K(p) C(p) \tilde{\Phi}\left(p, t_{0}\right) \mathrm{d} p .
$$

This equation holds for any pair of real numbers $\left(t, t_{0}\right)$, which can be replaced by any other pairs, say $(s, t)$,

$$
\tilde{\Phi}(s, t)-\Phi(s, t)=-\int_{t}^{s} \Phi(s, p) K(p) C(p) \tilde{\Phi}(p, t) \mathrm{d} p .
$$

Left multiply both sides by $C(s)$ and right multiply by any (arbitrary) unit vector $v \in \mathbb{R}^{n}$, and take the integral of the squared norm of both sides, then

$$
\begin{aligned}
& \int_{t-\sigma}^{t}\|C(s)(\tilde{\Phi}(s, t)-\Phi(s, t)) v\|^{2} \mathrm{~d} s \\
& =\int_{t-\sigma}^{t}\left\|C(s) \int_{s}^{t} \Phi(s, p) K(p) C(p) \tilde{\Phi}(p, t) v \mathrm{~d} p\right\|^{2} \mathrm{~d} s .
\end{aligned}
$$

Develop the squared Euclidean norm at the left hand side and rearrange the terms, then

$$
\begin{aligned}
& \int_{t-\sigma}^{t}\|C(s) \Phi(s, t) v\|^{2} \mathrm{~d} s=-\int_{t-\sigma}^{t}\|C(s) \tilde{\Phi}(s, t) v\|^{2} \mathrm{~d} s \\
& +\int_{t-\sigma}^{t} 2 v^{T} \Phi^{T}(s, t) C^{T}(s) C(s) \tilde{\Phi}(s, t) v \mathrm{~d} s \\
& +\int_{t-\sigma}^{t}\left\|C(s) \int_{s}^{t} \Phi(s, p) K(p) C(p) \tilde{\Phi}(p, t) v \mathrm{~d} p\right\|^{2} \mathrm{~d} s .
\end{aligned}
$$

Each of the terms involved in (18) will be examined in the following in order to derive an inequality bounding the observability Gramian. 
As $\|v\|=1$ (unit vector), it follows from (5) that

$$
\begin{aligned}
\alpha & =v^{T} \alpha I_{n} v \leq v^{T} M(t-\sigma, t) v \\
& =\int_{t-\sigma}^{t}\|C(s) \Phi(s, t) v\|^{2} \mathrm{~d} s \leq v^{T} \beta I_{n} v=\beta,
\end{aligned}
$$

therefore

$$
\alpha \leq \int_{t-\sigma}^{t}\|C(s) \Phi(s, t) v\|^{2} \mathrm{~d} s \leq \beta .
$$

Define

$$
\chi \triangleq \int_{t-\sigma}^{t}\|C(s) \tilde{\Phi}(s, t) v\|^{2} \mathrm{~d} s=v^{T} \tilde{M}(t-\sigma, t) v .
$$

By the Schwarz inequality,

$$
\begin{aligned}
& \int_{t-\sigma}^{t} 2 v^{T} \Phi^{T}(s, t) C^{T}(s) C(s) \tilde{\Phi}(s, t) v \mathrm{~d} s \\
& \leq 2 \sqrt{\int_{t-\sigma}^{t}\|C(s) \Phi(s, t) v\|^{2} \mathrm{~d} s \int_{t-\sigma}^{t}\|C(s) \tilde{\Phi}(s, t) v\|^{2} \mathrm{~d} s} \\
& \leq 2 \sqrt{\beta \chi}
\end{aligned}
$$

where the last inequality is based on (19) and (20).

The assumption that $\|A(t)\| \leq \gamma$ implies (see Lemma 3 )

$$
\|\Phi(s, p)\| \leq e^{\gamma|s-p|} .
$$

Notice that in the inner integral of the last term of (18) $t-\sigma \leq s \leq p \leq t$, then

$$
\begin{aligned}
\int_{s}^{t}\|\Phi(s, p)\|^{2} \mathrm{~d} p & \leq \int_{s}^{t} e^{2 \gamma(p-s)} \mathrm{d} p \\
& =\frac{1}{2 \gamma}\left(e^{2 \gamma(t-s)}-1\right) .
\end{aligned}
$$

Replace $\|C(s)\|$ and $\|K(p)\|$ by their respective upper bounds and apply the Schwarz inequality to the inner integral of the last term of (18), then

$$
\begin{aligned}
& \int_{t-\sigma}^{t}\left\|C(s) \int_{s}^{t} \Phi(s, p) K(p) C(p) \tilde{\Phi}(p, t) v \mathrm{~d} p\right\|^{2} \mathrm{~d} s \\
& \leq \eta^{2} \rho^{2} \int_{t-\sigma}^{t} \int_{s}^{t}\|\Phi(s, p)\|^{2} \mathrm{~d} p \int_{s}^{t}\|C(p) \tilde{\Phi}(p, t) v\|^{2} \mathrm{~d} p \mathrm{~d} s \\
& \leq \eta^{2} \rho^{2} \int_{t-\sigma}^{t} \frac{1}{2 \gamma}\left(e^{2 \gamma(t-s)}-1\right) \chi \mathrm{d} s \\
& =\eta^{2} \rho^{2}\left(\frac{1}{4 \gamma^{2}}\left(e^{2 \gamma \sigma}-1\right)-\frac{\sigma}{2 \gamma}\right) \chi .
\end{aligned}
$$

It then follows from (18), (19), (20), (21) and (25) that

$$
\alpha \leq-\chi+2 \sqrt{\beta \chi}+\varphi \chi
$$

with

$$
\varphi \triangleq \eta^{2} \rho^{2}\left(\frac{e^{2 \gamma \sigma}-1}{4 \gamma^{2}}-\frac{\sigma}{2 \gamma}\right) .
$$

Treat (26) as a polynomial inequality in terms of $\sqrt{\chi}$, of second degree if $\varphi \neq 1$, or of first degree otherwise.
Some straight-forward computations then show that

$$
\chi \geq\left\{\begin{array}{cl}
\left(\frac{\sqrt{\beta-\alpha+\varphi \alpha}-\sqrt{\beta}}{\varphi-1}\right)^{2}>0 & \text { if } \varphi \neq 1 \\
\frac{\alpha^{2}}{4 \beta}>0 & \text { if } \varphi=1
\end{array}\right.
$$

As $\chi$ is defined in (20) with an arbitrary unit vector $v \in \mathbb{R}^{n}$, the result expressed in (28) provides a lower bound of $\tilde{M}(t-\sigma, t)$, namely $\tilde{\alpha}$ as stated in Lemma 2 .

The upper bound of $\tilde{M}(t-\sigma, t)$ will be established similarly. Due to the "symmetry" between $A(t)$ and $\tilde{A}(t)$ expressed in (12) and (13), the "symmetric" counterpart of (18) corresponds to interchanging $\Phi(s, t)$ and $\tilde{\Phi}(s, t)$ and replacing $K(p)$ by $-K(p)$. It results in

$$
\begin{aligned}
& \int_{t-\sigma}^{t}\|C(s) \tilde{\Phi}(s, t) v\|^{2} \mathrm{~d} s=-\int_{t-\sigma}^{t}\|C(s) \Phi(s, t) v\|^{2} \mathrm{~d} s \\
& +\int_{t-\sigma}^{t} 2 v^{T} \tilde{\Phi}^{T}(s, t) C^{T}(s) C(s) \Phi(s, t) v \mathrm{~d} s \\
& +\int_{t-\sigma}^{t}\left\|C(s) \int_{s}^{t} \tilde{\Phi}(s, p) K(p) C(p) \Phi(p, t) v \mathrm{~d} p\right\|^{2} \mathrm{~d} s .
\end{aligned}
$$

Interchange the positions of the left hand side term and the first term of the right hand side, then, by reminding (19),

$$
\begin{aligned}
\alpha \leq & \int_{t-\sigma}^{t}\|C(s) \Phi(s, t) v\|^{2} \mathrm{~d} s=-\int_{t-\sigma}^{t}\|C(s) \tilde{\Phi}(s, t) v\|^{2} \mathrm{~d} s \\
& +\int_{t-\sigma}^{t} 2 v^{T} \tilde{\Phi}^{T}(s, t) C^{T}(s) C(s) \Phi(s, t) v \mathrm{~d} s \\
& +\int_{t-\sigma}^{t}\left\|C(s) \int_{s}^{t} \tilde{\Phi}(s, p) K(p) C(p) \Phi(p, t) v \mathrm{~d} p\right\|^{2} \mathrm{~d} s
\end{aligned}
$$

Like in the case of (21), the Schwarz inequality is applied to the second term of the right hand side of (29). The inequality

$$
\|\tilde{\Phi}(s, p)\| \leq e^{(\gamma+\eta \rho)|s-p|}
$$

is derived from Lemma 3. By treating the last term of (29) in the same manner as the last term of (18), then (29) leads to

$$
\alpha \leq-\chi+2 \sqrt{\beta \chi}+\psi \beta
$$

with

$$
\psi \triangleq \eta^{2} \rho^{2}\left(\frac{e^{2(\gamma+\eta \rho) \sigma}-1}{4(\gamma+\eta \rho)^{2}}-\frac{\sigma}{2(\gamma+\eta \rho)}\right) .
$$

Treat (31) as a second degree polynomial inequality in terms of $\sqrt{\chi}$, it then yields

$$
\chi \leq(\sqrt{\beta-\alpha+\psi \beta}+\sqrt{\beta})^{2},
$$

establishing an upper bound of $\tilde{M}(t-\sigma, t)$, namely $\tilde{\beta}$ as stated in Lemma 2. 


\section{A more accurate bound estimation}

In the proof of Lemma 2, the lower bound was based on inequality (26), whereas the upper bound based on inequality (31). In fact, each of these two inequalities constrains both the lower and the upper bounds. In the previous section, the choice of ignoring the intersection of the solutions of the two inequalities was for the purpose of deriving lower and upper bounds in a relatively simple form. In what follows, a more accurate estimate of the two bounds will be established, by carefully taking into account this intersection.

Lemma 4 Under the same assumptions and with the same notations as in Lemma 2, an estimation of the lower and upper bounds $\tilde{\alpha}$ and $\tilde{\beta}$ as defined in (7) is given as follows, exhaustively in four different situations (notice that $0 \leq \varphi \leq \psi$ always hold, following the definitions (10) and (11)):

if $0 \leq \varphi \leq \psi<\frac{\alpha}{\beta}<1$, then

$$
\begin{aligned}
\tilde{\alpha}= & \max \left[\left(\frac{\sqrt{\beta}-\sqrt{\beta-(1-\varphi) \alpha}}{1-\varphi}\right)^{2},\right. \\
& \left.(\sqrt{\beta}-\sqrt{\beta+\psi \beta-\alpha})^{2}\right] \\
\leq & \tilde{\beta}=\min \left[\left(\frac{\sqrt{\beta}+\sqrt{\beta-(1-\varphi) \alpha}}{1-\varphi}\right)^{2},\right. \\
& \left.(\sqrt{\beta}+\sqrt{\beta+\psi \beta-\alpha})^{2}\right]
\end{aligned}
$$

if $0 \leq \varphi<1, \frac{\alpha}{\beta} \leq \psi$ and $\varphi \leq \psi$, then

$$
\begin{aligned}
\tilde{\alpha}= & \left(\frac{\sqrt{\beta}-\sqrt{\beta-(1-\varphi) \alpha}}{1-\varphi}\right)^{2} \\
\leq & \tilde{\beta}=\min \left[\left(\frac{\sqrt{\beta}+\sqrt{\beta-(1-\varphi) \alpha}}{1-\varphi}\right)^{2},\right. \\
& \left.(\sqrt{\beta}+\sqrt{\beta+\psi \beta-\alpha})^{2}\right]
\end{aligned}
$$

if $1=\varphi \leq \psi$, then

$$
\begin{aligned}
\tilde{\alpha} & =\frac{\alpha^{2}}{4 \beta} \\
& <\tilde{\beta}=(\sqrt{\beta}+\sqrt{\beta+\psi \beta-\alpha})^{2}
\end{aligned}
$$

if $1<\varphi \leq \psi$, then

$$
\begin{gathered}
\tilde{\alpha}=\left(\frac{-\sqrt{\beta}+\sqrt{\beta+(\varphi-1) \alpha}}{\varphi-1}\right)^{2} \\
\leq \tilde{\beta}=(\sqrt{\beta}+\sqrt{\beta+\psi \beta-\alpha})^{2} .
\end{gathered}
$$

Following the same symmetry remark as in the case of Lemma 2, the converse of Lemma 4 is also implied by the lemma itself.

\section{Proof of Lemma 4}

The proof will be based on inequalities (26) and (31), which are established in the proof of Lemma 2 under the same conditions as the present lemma.

The proof consists of 3 steps: the computations of the solution sets of inequalities (26) and (31) in the first two steps, then the combination of them in the last step.

Step 1: solution of inequality (26)

First notice that $\beta>\alpha>0$ and $\varphi \geq 0$ imply $4 \beta+$ $4(\varphi-1) \alpha>0$. This fact is useful for solving inequality $(26)$.

Treat (26) as a polynomial inequality in terms of $\sqrt{\chi}$. The solution is found in the following cases.

Case 1a: $\varphi>1$. It is straightforward to check

$$
\left(\frac{-\sqrt{\beta}+\sqrt{\beta+(\varphi-1) \alpha}}{\varphi-1}\right)^{2} \leq \chi
$$

Case 1b: $\varphi<1$. Similarly,

$$
\begin{aligned}
& \left(\frac{\sqrt{\beta}-\sqrt{\beta-(1-\varphi) \alpha}}{1-\varphi}\right)^{2} \\
& \quad \leq \chi \leq\left(\frac{\sqrt{\beta}+\sqrt{\beta-(1-\varphi) \alpha}}{1-\varphi}\right)^{2} .
\end{aligned}
$$

Case 1c: $\varphi=1$. More easily,

$$
\chi \geq \frac{\alpha^{2}}{4 \beta} .
$$

Step 2: solution of inequality (31)

Notice that $\beta-\alpha+\beta \psi>0$ always holds and treat (31) as a polynomial inequality in terms of $\sqrt{\chi}$, the solution is found in the following cases.

Case 2a: $\alpha-\psi \beta>0$. The solution is

$$
\begin{aligned}
& (\sqrt{\beta}-\sqrt{\beta+\psi \beta-\alpha})^{2} \\
& \quad \leq \chi \leq(\sqrt{\beta}+\sqrt{\beta+\psi \beta-\alpha})^{2} .
\end{aligned}
$$


Case 2b: $\alpha-\psi \beta \leq 0$. Similarly,

$$
0 \leq \chi \leq(\sqrt{\beta}+\sqrt{\beta+\psi \beta-\alpha})^{2} .
$$

\section{Step 3: combination of the two inequality solutions}

The lower and upper bounds $\tilde{\alpha}$ and $\tilde{\beta}$ as defined in (7) are derived by taking the intersection of the two solution sets bounding $\chi$ in the two previous steps. To this end, the following facts will be used to ensure that $\tilde{\alpha} \leq \tilde{\beta}$ :

if $0 \leq \varphi \leq \psi<\frac{\alpha}{\beta}<1$, then

$$
\begin{aligned}
& \frac{\sqrt{\beta}-\sqrt{\beta-(1-\varphi) \alpha}}{1-\varphi} \leq \sqrt{\beta}+\sqrt{\beta+\psi \beta-\alpha} \\
& \sqrt{\beta}-\sqrt{\beta+\psi \beta-\alpha} \leq \frac{\sqrt{\beta}+\sqrt{\beta-(1-\varphi) \alpha}}{1-\varphi}
\end{aligned}
$$

if $0 \leq \varphi<1, \frac{\alpha}{\beta} \leq \psi$ and $\varphi \leq \psi$, then

$$
\frac{\sqrt{\beta}-\sqrt{\beta-(1-\varphi) \alpha}}{1-\varphi} \leq \sqrt{\beta}+\sqrt{\beta+\psi \beta-\alpha} ;
$$

if $1=\varphi \leq \psi$, then

$$
\frac{\alpha}{2 \sqrt{\beta}}<\sqrt{\beta}+\sqrt{\beta+\psi \beta-\alpha}
$$

if $1<\varphi \leq \psi$, then

$$
\frac{-\sqrt{\beta}+\sqrt{\beta+(\varphi-1) \alpha}}{\varphi-1} \leq \sqrt{\beta}+\sqrt{\beta+\psi \beta-\alpha} .
$$

The proof of these inequalities involves only tedious elementary mathematics.

Based on these results, the combination of Steps 1 and 2 lead to the lower and upper bounds $\tilde{\alpha}$ and $\tilde{\beta}$ as summarized in Lemma 4.

\section{A numerical example}

Consider the example

$A=\left[\begin{array}{llll}0 & 1 & 0 & 0 \\ 0 & 0 & 1 & 0 \\ 0 & 0 & 0 & 1 \\ 0 & 0 & 0 & 0\end{array}\right], C=\left[\begin{array}{llll}1 & 1 & 1 & 1 \\ 0 & 1 & 0 & 0 \\ 0 & 0 & 1 & 1\end{array}\right], K=\left[\begin{array}{lll}1 & 1 & 1 \\ 1 & 1 & 1 \\ 1 & 1 & 0 \\ 1 & 0 & 0\end{array}\right], \sigma=1$.

The lower and upper bounds of the symmetric positive definite matrix $M(t-1, t)$ are taken as its smallest and largest eigenvalues $\alpha=0.1160, \beta=2.8384$. The constants defined in (8) are $\gamma=1, \eta=2.3244, \rho=2.8092$. According to Lemma 2, the lower and upper bounds of $\tilde{M}(t-1, t)$ in this example are respectively $6.5340 \times$ $10^{-4} I_{4}$ and $1.8555 \times 10^{6} I_{4}$.

The bounds of (Ioannou and Sun, 1996, p. 226) are a revised version of those of (Sastry and Bodson, 1989, p. 73). After a correction of the bounds of (Ioannou and Sun, 1996) suggested by the Associate Editor in charge of the present paper (see Appendix A), the lower and upper bounds of $\tilde{M}(t-1, t)$ are respectively $4.2289 \times 10^{-4} I_{4}$ and $6.9497 \times 10^{119} I_{4}$.

\section{Conclusion}

This paper presented a complete proof of a classical result on the bounds of observability Gramian. This result is useful for the analysis of some adaptive systems. Two estimations of the bounds of the closed loop system observability Gramian are presented, at different levels of complexity and accuracy.

\section{A Discussion on existing results}

In (Sastry and Bodson, 1989, p. 73), Lemma 2.5.2 is about the relation between the matrix pairs $[A(t), C(t)]$ and $[A(t),(A(t)+K(t) C(t))]$, which is equivalent to the case considered in this paper, up to the sign of $K(t)$. The proof of Lemma 2.5.2 on page 332 starts by considering the two systems

$$
\begin{gathered}
\dot{x}(t)=A(t) x(t) \\
y(t)=C(t) x(t) \\
\dot{w}(t)=(A(t)+K(t) C(t)) w(t) \\
z(t)=C(t)) w(t)
\end{gathered}
$$

with respectively their associated state transition matrices $\Phi\left(t, t_{0}\right)$ and $\bar{\Phi}\left(t, t_{0}\right)$. The authors then introduce the unit vector

$$
e(s) \triangleq \frac{K(s) C(s) w(s)}{\|K(s) C(s) w(s)\|}
$$

which is required in the following part of the proof. However, $e(s)$ is not well defined when $\|K(s) C(s) w(s)\|=0$. This situation can indeed happen for $w(s) \neq 0$ if the matrix $K(s) C(s)$ is rank deficient. In particular, $e\left(t_{0}\right)$ is not well defined when the initial state $w\left(t_{0}\right)$ satisfies $K\left(t_{0}\right) C\left(t_{0}\right) w\left(t_{0}\right)=0$.

Moreover, the following part of the proof looks for upper and lower bounds of

$$
\begin{aligned}
& \int_{t_{0}}^{t_{0}+\sigma}\left\|C(\tau) \bar{\Phi}\left(\tau, t_{0}\right) w\left(t_{0}\right)\right\|^{2} \mathrm{~d} \tau \\
& =w^{T}\left(t_{0}\right)\left(\int_{t_{0}}^{t_{0}+\sigma} \bar{\Phi}^{T}\left(\tau, t_{0}\right) C^{T}(\tau) C(\tau) \bar{\Phi}\left(\tau, t_{0}\right) \mathrm{d} \tau\right) w\left(t_{0}\right)
\end{aligned}
$$

for all unit vector $w\left(t_{0}\right) \in \mathbb{R}^{n}$, where $\bar{\Phi}\left(\tau, t_{0}\right)$ is the state transition matrix associated to $A(t)+K(t) C(t)$, hence it is impossible to avoid the case of $K(s) C(s) w(s)=0$ when $K(s) C(s)$ is rank deficient.

In (Ioannou and Sun, 1996, p. 226), the authors tried to remedy this problem with

$$
e(s) \triangleq \begin{cases}\frac{K(s) C(s) w(s)}{\|K(s) C(s) w(s)\|} & \text { if }\|C(s) w(s)\| \neq 0 \\ \frac{K(s)}{\|K(s)\|} & \text { if }\|C(s) w(s)\|=0 .\end{cases}
$$

Notice that $e(s)$ is an $n \times m$ matrix in the second case of this new definition, whereas $e(s)$ was always a vector in (A.3). One of the steps of the proof (see (Ioannou and Sun, 1996, p. 226), the line of two inequalities before 
$(4.8 .10))$ is based on the two inequalities

$$
\begin{aligned}
\int_{t_{0}}^{t_{0}+\sigma}\|C(\tau) \Phi(\tau, s) e(s)\|^{2} \mathrm{~d} \tau & \leq \int_{t_{0}}^{t_{0}+\sigma}\|C(\tau) \Phi(\tau, s)\|^{2} \mathrm{~d} \tau \\
& \leq \beta_{2},
\end{aligned}
$$

where $\beta_{2}$ is a constant such that

$$
\int_{t_{0}}^{t_{0}+\sigma} \Phi^{T}(\tau, s) C^{T}(\tau) C(\tau) \Phi(\tau, s) \mathrm{d} \tau \leq \beta_{2} I_{n} .
$$

Unfortunately, inequality (A.5) is incorrect, as shown by the counter example

$$
A=\left[\begin{array}{ll}
0 & 1 \\
0 & 0
\end{array}\right], \quad C=\left[\begin{array}{ll}
1 & 0
\end{array}\right], s=0, \quad t_{0}=0, \quad \sigma=1 .
$$

It can be checked that $\beta_{2}=\frac{\sqrt{13}}{6}+\frac{2}{3}$ satisfies (A.6), but

$$
\int_{t_{0}}^{t_{0}+\sigma}\|C(\tau) \Phi(\tau, s)\|^{2} \mathrm{~d} \tau=\frac{4}{3}>\beta_{2} .
$$

Following a suggestion by the Associate Editor in charge of this paper, the incorrect bound $\beta_{2}$ can be replaced by

$$
\beta_{2}^{*}=\eta^{2} \frac{e^{2 \gamma \sigma}-1}{2 \gamma}
$$

Nevertheless, the modified definition of $e(s)$ in (A.4) does not fully avoid the problem of division by zero, as $\|C(s) w(s)\| \neq 0$ does not prevent that $\|K(s) C(s) w(s)\|=0$. The correction with $\beta_{2}^{*}$ was for the mistake of inequality (A.5), it does not repair this problem of possible division by zero.

Acknowledgement. The authors would like to thank the associate editor and the reviewers for their comments that have helped to improve this paper.

\section{References}

Aeyels, D., Sepulchre, R., and Peuteman, J. (1998). Asymptotic stability for time-variant systems and observability: Uniform and nonuniform criteria. Mathematics of Control Signals and Systems, 11(1):1-27.

Anderson, B. D., Bitmead, R. R., Johnson, C. R. J., Kokotovic, P. V., Kosut, R. L., Mareels, I. M., Praly, L., and Riedle, B. D. (1986). Stability of adaptive systems: passivity and averaging analysis. Signal processing, optimization, and control. MIT Press, Cambridge, Massachusetts.

Chicone, C. (2006). Ordinary Differential Equations with Applications. Springer, New York.

Ioannou, P. and Sun, J. (1996). Robust Adaptive Control. Prentice Hall. Electronic copy http://wwwrcf.usc.edu/ ioannou/Robust_Adaptive_Control.htm.

Kalman, R. E. (1963). New methods in Wiener filtering theory. In Bogdanoff, J. L. and Kozin, F., editors, Proceedings of the First Symposium on Engineering Applications of Random Function Theory and Probability, New York. John Wiley \& Sons.

Sastry, S. and Bodson, M. (1989). Adaptive Control: Stability, Convergence and Robustness. PrenticeHall. 